\title{
Thermal effects in dry sliding contacts
}

by G. Cuccurullo, V. Spingi, R. Di Giuda

*Dept. of Industrial Engineering, University of Salerno, Via Ponte Don Melillo - 84084 Fisciano (SA), Italy, cuccuru@unisa.it

\begin{abstract}
The tribological behaviour of the friction disk and the associated counter faces depends significantly on their dynamic contact temperature. Heat generated by friction at the contact surfaces is shared between them and determines the related temperature rise which has to be estimated for operating and designing the engagement in stable conditions. In this paper, first, an easy-to-handle 2D analytical model is proposed to take into account the partition of the generated heat between the coupled surfaces and, as a consequence, to determine the related temperature rise. Then, considering that measuring temperatures in a kinematical pair is a difficult task, a procedure for catching the proper correlation between the friction coefficient and the interface temperatures during actual friction braking tests is proposed. Preliminary experiments were satisfyingly realized for selected values of the main operating parameters, such as disk speed and braking load. The analytical model response was validated through a commercial FEM code, thus assuring the correctness of the procedure.
\end{abstract}

\section{Introduction}

The clutch tribological behaviors depend significantly on the dynamic contact temperature of the frictional surfaces, which in turn is related to normal pressure load, relative sliding velocity and the nature of the involved surfaces. The interface temperature has momentous influence on friction and wear effects, with potential failures caused by high thermal levels and thermomechanical stresses. It is well known that the temperature sensitivity of friction materials is a critical aspect wishing to ensure their smooth and reliable functioning. In fact, it influences the thermo-elastic instabilities, which in turn alter the friction performance at the braking junctions [1]. In the front wheel, for example, disk brake pads absorb a major amount (up to $80 \%$ ) of the total kinetic energy of an automobile. This causes the generation of high temperature up to $370^{\circ} \mathrm{C}$ on the disk. The severity of such temperature rise is further manifested in the form of a very high flash temperature up to $600^{\circ} \mathrm{C}$ at the contacting asperities [2]. At such high temperatures, pads suffer from a loss of effectiveness called "fade" because of a reduction in the kinetic friction coefficient. High interfacial temperatures can lead to a decrease in shear strength of the pad and consequently a decrease in frictional force which induces fade [3]. Thus, correlating the friction coefficient to the operating temperature level for the coupled friction surfaces is probably the most critical point in designing safe and effective braking systems and it is not surprising that many researchers pay their attention to theoretical models for surface temperature evaluation as well as to surface temperature measurements. A lot of studies about heat transfer at the coupled interfaces are reported in literature which take into account the superposition of several phenomena, each described by a number of simplifying assumptions: experimental, analytical and numerical methods are attempted to understand and to predict wear effects. From an experimental standpoint, the system relative motion, its geometry and the high thermal gradients which are typically attained make experimental temperature detection a complex matter if one wishes to use thermocouples located directly at the friction interface $[4,5,6]$. Therefore, "non-contact measurements" including optical or infrared methods could be the solution and early works are appearing in this sense $[7,8,9,10]$. The latter technique seems to be the most effective and valuable due to its ability to perform continuous temperature map recording with relatively high resolutions when compared to other traditional methods but it is to consider that the area to focus on is "hidden" by the coupled surfaces. Due to the described difficulties in the experimental approach, in recent years, the finite element method [11] has become the preferred method for studying the problem at hand while few analytical models are also available [12]. However, a critical analysis of the above mentioned works allows to clearly conclude that, often, numerical predictions for temperature distribution aren't adequately validated by experimental tests.

In the above connection, a two-dimensional analytical model is proposed for describing the temperature field and the partition of heat for a moving source which is in rectilinear motion. The latter configuration allows a relatively simple solution from the analytical point of view: it is based on trigonometric functions whereas more complicated solutions, such as the ones cited before, are available. Notwithstanding, carrying on numerical runs on a commercial FEM code, it was proven that, temperature field arising from circular motion is fully recovered by the model at hand, provided suitable operative conditions are realized. Thus, an easy to handle procedure is proposed aiming to catch the proper correlation between the friction coefficient and the interface temperatures during actual friction braking tests, for selected values of operating parameters, such as speed and braking load.

For the purposes of applying the above, experiments were performed on a specifically designed pin-on-disc device which allowed to overcome the addressed uncertainty due to the transducer positioning: temperature data were collected by means of infrared thermography looking outside of the optically-hidden contact area. Then, a proper data 


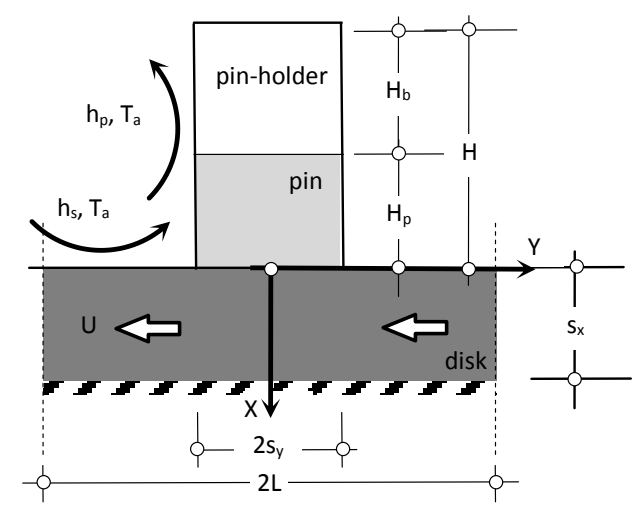

Fig. 1. Schematic of the analytical problem

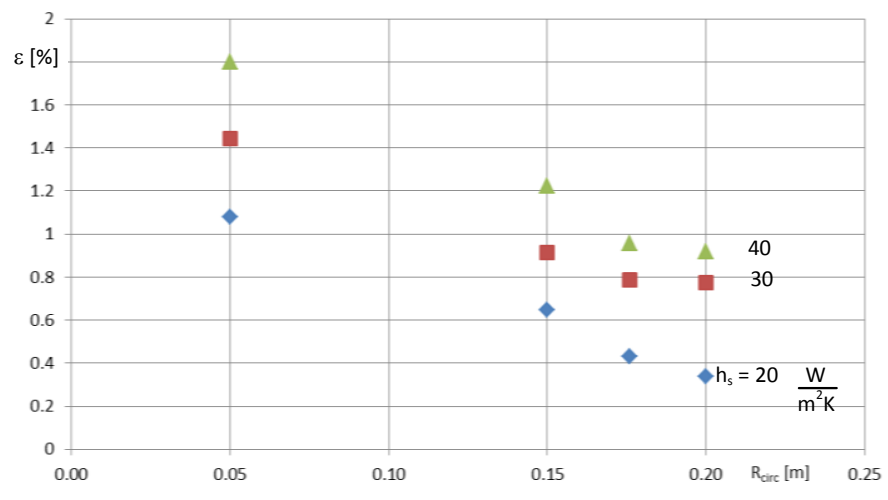

Fig. 2. numerical validation of the analytical solution for adiabatic surfaces with the exception of the pin path

reduction based on the two-dimensional model described in the following section allowed to reconstruct the average temperature in the hidden area. At the same time, the friction coefficient was measured using two load cells; the former was employed to measure the normal load, the latter to evaluate the tangential force. Whereas standard laboratory tests are available for featuring wear phenomena, they are long-lasting, highly expensive and, most of all, they scarcely refer details about temperature distribution $[13,14,15,16]$ for the samples under test.

\section{Basic equations}

A 2D theoretical model to predict the temperature rise due to a moving heat source was proposed by the same authors of the present work in a companion paper [17 in press]. The scheme on which the model was based is shown in figure 1. A finite thickness slab moving at a constant velocity $U$ in the y-direction is in contact with a pin. An uneven distribution for heat generation at the interface was allowed to heat the slab which, at the same time, was cooled by radiative-convective heat transfer (featured by the coefficient $h_{\mathrm{s}}$ ) to an ambient at uniform temperature. The slab was assumed adiabatic on its lower surface while it was subjected to thermal coupling conditions on the remaining edges $( \pm L)$. The pin was cooled on its lateral surface by radiative-convective heat transfer (featured by the coefficient $h_{p}$ ) and it was described involving the thin rod model, since its transversal Biot number is generally very low for the application at hand. In order to account for the pin-holder effect, a further thin rod is considered in series with respect to the pin which is in perfect thermal contact with it and long enough to be considered adiabatic on the free end. Thus, heat transfer is two-dimensional in the slab and one-dimensional in the remaining domain.

For such a problem, if the observer is moving on with the pin and if a suitable time has elapsed, the energy balance equation and the related boundary conditions for the slab turn out to be time-independent. For such a configuration, the dimensionless energy balance equation for the disk was solved analytically in closed form by the Fourier series; in particular the structure for the temperature field was sought to be:

$$
\vartheta(\xi, \eta)=\sum_{k=-\infty}^{\infty} Q_{k}(\xi) e^{i \Omega k \eta}
$$

where: $\xi=X / s_{\mathrm{x}}, \eta=Y / s_{\mathrm{x}}, \theta=\left(T-T_{\mathrm{a}}\right) /\left(s_{\mathrm{x}} q_{\mathrm{s}} / k_{\mathrm{s}}\right), k_{\mathrm{s}}$ being the slab conductivity and $T_{\mathrm{a}}$ the surrounding temperature; $\Omega=$ $s_{\mathrm{x}} \pi d L$ is the spatial frequency, $\omega=s_{\mathrm{x}} U /(2 \alpha)$ is the Peclet number, $\alpha$ being the slab diffusivity. The heat flux entering the slab though the contact area is assumed to be described such as $q_{\mathrm{slab}}(Y)=q_{\mathrm{s}} f(Y)$, where $q_{\mathrm{s}}$ is the unknown intensity heat flux entering into the slab and $f(Y)$ represents its spatial distribution which, for the present purposes, is assumed to be uniform: $f(Y)=1$. Finally, $B i_{\mathrm{s}}=h_{\mathrm{s}} S_{\mathrm{x}} / k_{\mathrm{s}}$ is the Biot number of the slab. The unknown $Q_{\mathrm{k}}(\xi)$ functions are determined by solving the following problem:

$$
Q_{\mathrm{k}}^{\prime \prime}+\left(2 \omega i \Omega k-\Omega^{2} k^{2}\right) Q_{\mathrm{k}}=0
$$

$$
Q_{\mathrm{k}}{ }^{\prime}(0)=B i Q_{\mathrm{k}}(0)-F_{\mathrm{k}} \quad Q_{\mathrm{k}}{ }^{\prime}(1)=0
$$

Then the local dimensionless temperature for the slab $\theta$ turns out to depend on the Peclet number and on the Biot number of the slab.

The heat conduction in the pin and in the pin-holder is assumed to be one-dimensional. The above hypothesis is realistic as much as the transverse Biot number is small which typically is the case for the systems at hand. Finally, the 


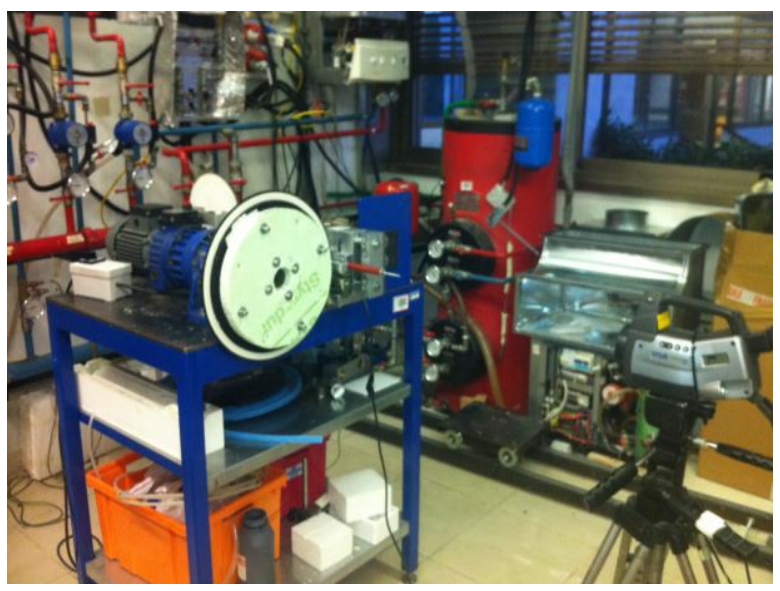

Fig. 3. The test rig

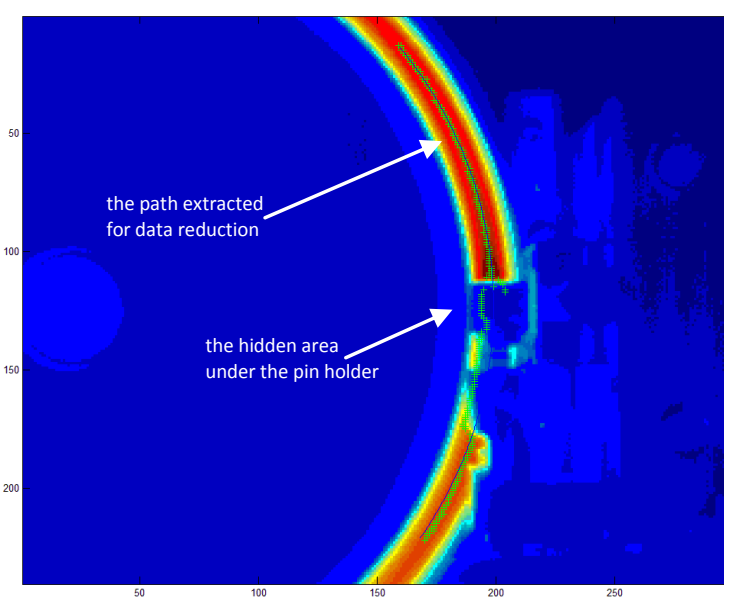

Fig. 4. A typical infrared image of the pin thermal track

1D dimensionless energy equations for the pin (subscript "p") and the pin-holder (subscript "b") were written to obtain the related analytical solution, depending on the geometry, boundary conditions and materials involved.

Both for the pin and for the slab, the average temperature under the contact area, $T_{\mathrm{av}}$, was related to the corresponding thermal resistances, $R_{\mathrm{pb}}$ and $R_{\mathrm{s}}$ :

$$
T_{\mathrm{av}}-T_{\mathrm{a}}=R_{\mathrm{pb}} q_{\mathrm{p}} \quad T_{\mathrm{av}}-T_{\mathrm{a}}=R_{\mathrm{s}} q_{\mathrm{s}}
$$

$q_{p}$ and $q_{s}$ being the heat flux entering the pin and the slab, respectively; perfect contact between the pin and the slab, was assumed. If the conservation of energy @ the interface is written as $q_{\mathrm{g}}=q_{\mathrm{s}}+q_{\mathrm{p}}, q_{\mathrm{g}}$ being the overall energy generated by friction, then the heat partition coefficient, $p_{\mathrm{s}}$, between the pin and the slab is determined as:

$$
p_{\mathrm{s}}=\frac{q_{\mathrm{s}}}{q_{\mathrm{g}}}=\frac{R_{\mathrm{pb}}}{R_{\mathrm{pb}}+R_{\mathrm{s}}}
$$

The remarkable role of the partition index parameter stems from considering that the heat generated by friction is divided between the pin and the disc as a function of the materials' properties and the heat exchange with the environment; since this partition linearly influences both the disk and the pin temperature levels, it is one of the main responsible parameter in determining the temperature field for the coupled surface under frictional conditions.

\section{Validation of the analytical solution}

The validation of the proposed analytical solution is done [17 in press] through the comparison of the temperature patterns on the frictional surface evaluated by a commercial FEM code, Comsol, $T^{*}$, with the model under study, $T$. Numerical modelling encompassed a rectangular $(12 \mathrm{~mm}$ radial $\times 20 \mathrm{~mm}$ tangential lenght) heat source moving along a circular path of radius $R_{\text {circ }}$ in order to approach more closely the typical geometries featuring the pin-on-disk devices. A first comparison was carried out considering the pin running on a circumference with infinite radius $R_{\text {circ. }}$ The parameter used to evaluate the difference between the numerical and analytical solutions was the relative error between the maximum temperatures on the contact area, $\varepsilon=\left(T_{\max }-T_{\max }^{*}\right) / T_{\text {max }}^{*}$. The relative error $\varepsilon$ between the two models always remained less than $1 \%$ for different speeds, geometries and boundary conditions, thus assuring the correctness of the analytical solution.

Having in mind to use the analytical model as the core for the procedure outlined in the next paragraph and aiming to recover as close as possible the linear motion conditions in successive experiments, the disk was assumed to be adiabatic with the exception of the path covered by the pin. The move is valuable in order to reduce the conduction normal to the $X Y$ plane (see figure 1). Moreover, it allows to determine temperature rise under the contact area in safe design conditions. The relative error was then evaluated assuming a conceivable range for both the heat transfer coefficients and thermal conductivities involved in the experimental testing introduced later. Assuming the set of parameters reported in paragraph 5 to be fixed, it is shown in figure 2 that the error $\varepsilon$ is always less than $2 \%$ and it decreases with increasing the pin-path radius for a fixed heat transfer slab coefficient. As expected, for a fixed radius $R_{\text {circ }}$, the error reduces for decreasing $h_{\mathrm{s}}$. Thus, it has been proven that the numerically calculated circumferential temperature profiles are fully recovered by the analytical model for the prescribed operative conditions. 
Table 1. Data reduction

\begin{tabular}{|c|c|r|r|r|r|r|r|}
\hline$F_{\mathrm{N}}$ & $\mathrm{U}$ & \multicolumn{1}{c|}{$\mathrm{k}$} & \multicolumn{1}{c|}{$\mathrm{h}_{\mathrm{s}}$} & \multicolumn{1}{c|}{$\mathrm{p}_{\mathrm{d}}$} & \multicolumn{1}{c|}{$\mathrm{T}_{\mathrm{av}}$} & $\mathrm{f}$ & \multicolumn{1}{c|}{ RMSE } \\
\hline$[\mathrm{N}]$ & {$[\mathrm{m} / \mathrm{s}]$} & {$[\mathrm{W} /(\mathrm{mK})]$} & {$\left[\mathrm{W} /\left(\mathrm{m}^{\wedge} 2 \mathrm{~K}\right)\right]$} & & {$\left[{ }^{\circ} \mathrm{C}\right]$} & & \\
\hline 174 & 0.54 & 0.372 & 27.35 & 0.9695 & 77.68 & 0.164 & $1.44 \mathrm{E}-05$ \\
\hline 87 & 0.27 & 0.371 & 20.96 & 0.9598 & 41.50 & 0.158 & $3.95 \mathrm{E}-05$ \\
\hline 174 & 0.27 & 0.360 & 22.50 & 0.966 & 54.78 & 0.157 & $1.99 \mathrm{E}-05$ \\
\hline 87 & 0.54 & 0.377 & 25.21 & 0.9677 & 53.28 & 0.165 & $1.35 \mathrm{E}-05$ \\
\hline
\end{tabular}

\section{Experimental setup}

A specifically designed pin on disk testing device was set up, figure 3. The wear rigs essentially consisted of two cylindrical pins in eccentric contact with a rotating disk. The two stationary pin specimens were symmetrically pressed against the disk. Disc rotation was allowed by an electric motor, an hydraulic speed regulator and a pulley-belt transmission. The rectangular pin (12 $\mathrm{mm}$ radial length, $20 \mathrm{~mm}$ circumferential length) was made of PTFE. The disc is made of bakelite ( $4 \mathrm{~mm}$ thickness, $36.6 \mathrm{~cm}$ maximum radius) and the aluminium pin-holder is $5 \mathrm{~cm}$ long. A spring device allowed to control the normal load $\left(F_{\mathrm{N}}\right)$. The spring load is imposed by a suitably configured dynamometer; the resulting toque is measured by a force gauge thus enabling to calculate the friction coefficient as the ratio between the latter to the former force.

Educated by the insights illustrated in the above paragraph, the disc surfaces were covered by sticking panels made up of a closed-cell insulating polymer matrix $3 \mathrm{~cm}$ thick. The insulated disk area encompassed all the exposed surfaces with the exception of that covered by the two pins in relative motion with respect to the disk. Provided a suitable emissivity calibration, the thermal pattern left on the disc by the track due to the relative pin motion was detected by an IR camera. The IR equipment (Thermacam Flir P65) exhibited a spatial resolution of about 30 dpi, due to $320 \times 240$ pixel matrix, the operating distance and optics in use. A typical infrared image is reported in figure 4; here, the pin track is clearly visible whereas it is possible to appreciate that the pin-holder covers the contact area. As a consequence, it was possible to detect temperatures along a track with the exclusion of an area $3 \mathrm{~cm}$ wide along the circumferential pin-track and placed around the pin itself. It can be argued that the presence of the pin-holder always interferes with optical measurements, however it was shown that a suitable data reduction can be done elaborating a proper collection of experimental data [18].

\section{Data reduction}

When performing measurements, it is assumed that temperature rise on the loaded surface is described by the temperature distribution given by the above model, after thermal equilibrium is attained. Due to the complexity of the response model, the Levenberg Marquadt technique based fitting method has been selected. The technique enables to process non-linear models with an arbitrary number of parameters. Thus, the optimal choice for matching experimental and theoretical data is accomplished by minimizing the RMSE merit function:

$$
R M S E=\frac{1}{N} \sum_{i=1}^{N}\left[\frac{T_{i}-T\left(y_{i}, \mathbf{a}\right)}{T_{a v}-T_{a}}\right]^{2}
$$

where the function $T(y, a)$ is the functional relationship given by the model for the disk surface temperature, $a=\left(h_{\mathrm{s}}, \mathrm{k}\right)$ being the unknown-parameters vector; the $N$ experimental data points, $\left(T_{i}, y_{i}\right)$, are detected by processing IR images; in particular, a suitably written code circumferentially scans the pin track seeking after the maximum temperature attained for each radius. The scan process is evidenced in Fig. 4: such points are then extracted and subjected to data reduction.

The remaining parameters influencing the slab temperature distribution are fixed as follows: for the disk, $\rho=$ $1300 \mathrm{~kg} / \mathrm{m}^{3}, c_{\mathrm{p}}=1465 \mathrm{~J} /(\mathrm{kgK})$; for the pin and pin holder, the thermal conductivities and lengths were $k_{\mathrm{p}}=0.21 \mathrm{~W} /(\mathrm{mK})$, $k_{\mathrm{b}}=160 \mathrm{~W} /(\mathrm{mK}), H_{\mathrm{p}}=5 \mathrm{~mm}, H_{\mathrm{b}}=50 \mathrm{~mm}$; for the ambient, $h_{\mathrm{p}}=10 \mathrm{~W} /\left(\mathrm{m}^{2} \mathrm{~K}\right) T_{\mathrm{a}}=23.8^{\circ} \mathrm{C}$. The friction coefficient is calculated by evaluating the imposed normal load and measured torque.

\section{Results and discussion}

Preliminary experimental tests were carried on by considering two different angular disk-speeds corresponding to peripheral values of $U_{1}=0.27 \mathrm{~m} / \mathrm{s}$ and $U_{2}=0.54 \mathrm{~m} / \mathrm{s}$ for the selected pin path radius, that is $17.6 \mathrm{~cm}$. Two pin-loads were considered, namely 87 and $174 \mathrm{~N}$. All the experiments were executed in triplicates.

The extracted circumferential thermal pattern were subjected to the data reduction procedure obtaining the average values reported in table 1 . It can be noted that the values obtained for the bakelite thermal conductivity are coherent with the ones declared, while the (average) heat transfer coefficients are almost the same for a fixed disk speed and increase with it. 


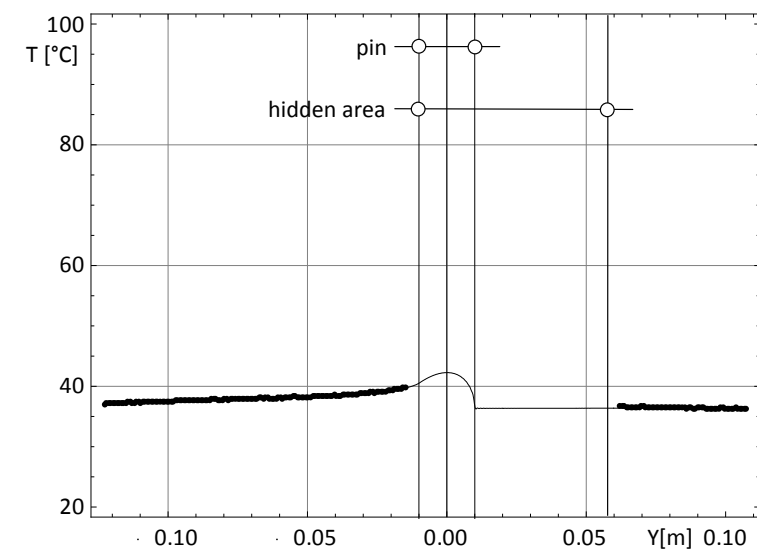

Fig. 5. Data reduction for $U=0.27 \mathrm{~m} / \mathrm{s}$ and $F_{\mathrm{N}}=87 \mathrm{~N}$

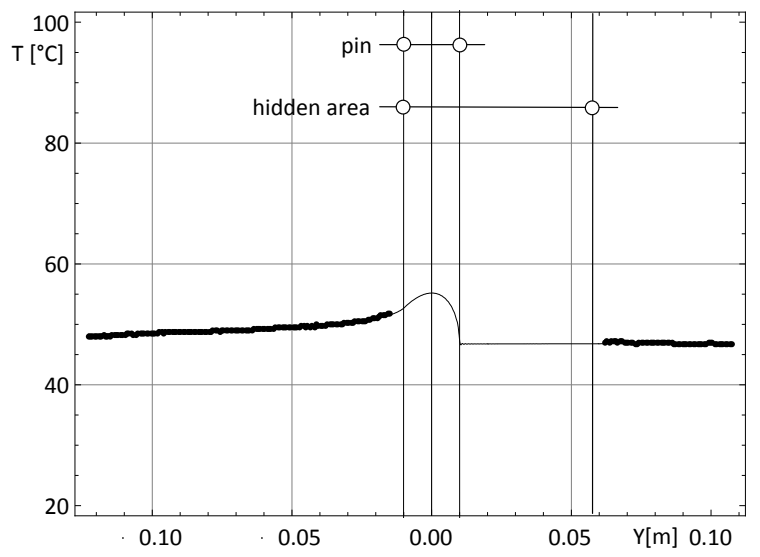

Fig. 7. Data reduction for $U=0.54 \mathrm{~m} / \mathrm{s}$ and $F_{\mathrm{N}}=87 \mathrm{~N}$

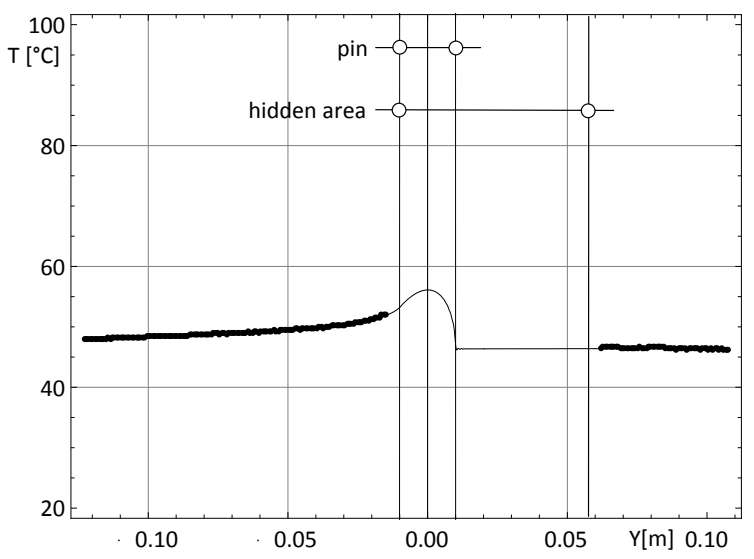

Fig. 6. Data reduction for $U=0.27 \mathrm{~m} / \mathrm{s}$ and $F_{\mathrm{N}}=174 \mathrm{~N}$

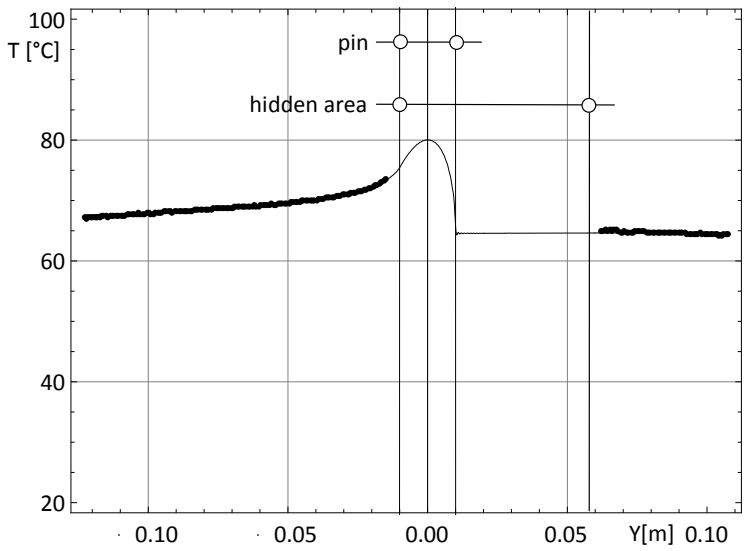

Fig. 8. Data reduction for $U=0.54 \mathrm{~m} / \mathrm{s}$ and $F_{\mathrm{N}}=174 \mathrm{~N}$

The values arising from the data reduction were used to build the analytical profiles reproduced in figures 5-8: the agreement with experimental points seems to be quite satisfying, as deducible from RMSE values.

It has to be underlined that the differences between the maximum values attained by temperature the under contact area and the nearest detectable temperatures are: $2.4,3.8,3.5$ and $6.6{ }^{\circ} \mathrm{C}$ for the plots reported in figures $5-8$, respectively. These values are expected to be amplified for increasing temperature levels. Thus, it is evident that the region under the contact area is a critical one due to the occurrence of high thermal gradients which could lead to get wrong temperature estimates. Finally, a check about f-values shows that they satisfyingly agree the expected dry friction characteristic [19]. Due to the narrow temperature range exploited in the present tests, it appears that the friction coefficient essentially depends on the disk speed [20].

\section{Conclusions}

An easy-to-handle analytical solution was employed to calculate the temperatures and the partition coefficient in a pin-on-disk device. It has been proven that the solution can avoid heavy three-dimensional numerical modelling while allowing satisfying predictions for typical operating conditions. It is simple to encode because the functions involved are well known. Once again, the pin on disk device has been proven to be the most effective and reliably device for determining friction and wear aspects; in addition, the experimental setup at hand allowed proper measurements for temperature rise based on the availability of an easy-to-handle analytical model and on IR temperature measurements. By running preliminary experiments according to the outlined procedure, a quite satisfying agreement with analytical predictions was realized, thus a reliable estimate has been obtained for the maximum temperature under the contact area, where direct measuring is always critical. The above results are then encouraging in extending experiments to 
encompass a wider temperature range thus aiming to seek for a proper correlation between the estimated temperature under the contact area and the friction coefficient in different operating conditions.

\section{REFERENCES}

[1] Bijwe J., Nidhi and Satapathy B. K. , "Influence of amount of resin on fade and recovery behaviour of nonasbestos organic (nao) friction materials", Trans. Indian Inst. Met.,vol.57, pp.335-344, 2004.

[2] Gopal P., Dharani L.R., Blum F.D. "Hybrid phenolic friction composites containing kevlar pulp: part I enhancement of friction and wear performance" Wear, vol.181, pp. 913-921, 1995.

[3] W. S. Scholtz "Brake pad technical advice", Article issued by Safeline, November 2002.

[4] J. Bijwe,Nidhi,N. Majumdar,B.K.Satapathy, "Influence of modified phenolic resins on the fade and recovery behavior of friction materials", Wear, vol. 259, pp. 1068-1078, 2005.

[5] Huang Feng, Mo Yimin, Lv Junnchering, "Study on heat feading of phenolic resin friction material for microautomobile clutch", International Conference on Measuring Technology and Mechatronics Automaition, 2010.

[6] H.S. Qi, "A contact length model for grinding whell- workpiece contact", PhD. Thesis, Liverpool Jhon Moores University, Uk, 2002.

[7] O.S.C. Dinc, M. Ettles, S.J. Calabrese, H.A. Scarton, "The measurement of surface temperature in dry or lubricated sliding", Trans. ASME, J. Tribol., vol. 115, 1993.

[8] Anon, "Material development using infra-red thermography", Metallurgia, vol. 62, 1995.

[9] G. Cuccurullo, V. D’Agostino, R. Di Giuda, A. Senatore, V. Spingi, An Analitycal Solution and an Experimental Approach for Thermal Field at the Interface of Dry Sliding Surfaces, Meccanica, Springer, vol. 46, pp. 589-595, ISSN 0025-6455, 2011.

[10] D. Majacherczak, P. Dufrenoy, Y. Berthier, "Tribological, thermal and mechanical coupling aspects of dry sliding contact", Tribology International, vol.40, pp. 834-843, 2007.

[11] L. Zhang, Q Yang, D. Weichert, N. Tan. "Simulation and Analysis of Thermal Fatigue Based on Imperfection Model of Brake Discs“, Beijing Jiaotong University, PAMM, Proc. Appl. Math. Mech., vol. 9, pp 533 - 534, 2009.

[12] N. Laraqui, N. Alilat, Gar J. M. cia De Maria, A. Bairi, "Termperature and division of heat in a pin-on-disk frictional device-exact analytical solution“, Wear, vol. 266, pp. 765-770, 2009.

[13] M. Bezzazi, A. Khamlichi , A. Jabbouri, P. Reis, J.P. Davim, "Experimental characterization of frictional behaviour of clutch facings using Pin-on-disk machine", Materials and Design, vol. 28 , pp. 2148-2153, 2007.

[14] RIP Test programs. R + D Department, Raybestos, Morbach (Germany), 1996.

[15] JIS D-4311: "Clutch facings for automobiles", Tokyo, Japanese Standards Association, 1995.

[16] G. Nicholson, "Facts about friction" Wincheste (Virginia), Gedoran America Limited, 2000.

[17] G. Cuccurullo, V. Spingi, R. Di Giuda, "An Analytical solution for heat partition in a pin-on-disc frictional device", XXX Uit Heat Transfer Conference, Bologna (Italy), June 25-27, 2012, in press.

[18] G. Cuccurullo, V. Spingi, "Thermographic inspection at the Interface of dry Sliding Surfaces", 17th Thermo, Scientific Society of Measurement, Automation and Informatics, Budapest, 2011.

[19] W. Gregory Sawyer, K.D. Freudenberg, P. Bhimaraj, L. Schadler, "A Study on the friction and wear behavior of PTFE filled with alumina nanoparticles", Wear, vol. 254, pp. 573-580, 2003.

[20] A. Senatore, V. D'Agostino, R. Di Giuda, V. Petrone, "Experimental Investigation and Neural Network prediction of brakes and clutch material frictional behavior considering the sliding acceleration influence", Tribology International, vol. 44, pp. 119-1207, ISSN:0301-679X, 2011. 